Zellen, d. h. die Anpassung an die Wachstumsreize, bewirken. Stehen wir auf dem Standpunkte, dass diese Anpassung nur dadurch zustande kommt, dass die wachstumserregenden Schädlichkeiten die für sie empfindlicheren Zellteile stärker lädieren, als die an und für sich widerstandsfähigeren, so dass letztere das Uebergewicht erlangen und die Zelle dadurch gegen die Reize weniger empfindlich wird, so können wir uns das beschriebene Verhalten in der Weise erklären, dass das Selbsthemmungsvermögen der Zellen gegen Wachstumsreizung um so eher verloren geht, je allgemeiner, komplexer die Läsion der Zellen ist, weshalb die Kombination zweier verschiedenartiger Reize, welche die verschiedensten Teile der Zellen schädigt, die Anpassung mehr erschwert, als die Anwendung einer einzigen oder zweier verhältnismässig verwandter Reizungen. Denken wir uns jedoch, dass für die Regulierung des Zellwachstums ein ähnliches selbständiges Organ existiert, wie wir es für die Zellteilung postuliert, so müssten wir annehmen, dass dieses Organ unter den verschiedenartigen Reizen auch in differentem Grade leidet, und zwar vor allem indirekt, durch die Schädigung anderer Zellteile geschwächt wird. Nur unter letzterer Voraussetzung kömnen wir es verstehen, dass die Kombination differenter Reize dieses Organ mehr schädigen soll, als eine solche verwandter Traumen.

Prinzipiell ist aber auch die Abnahme der Anpassungsfähigkeit nach Kombination verwandter Reize, also bei herabgeminderter Empfindlicbkeit der Gewebe von hohem Interesse. Die Hemmungen gegen die Wachstumsreize können - nach diesen Erfahrungen zu schliessen - steigen, während das Vermögen, sie zu verstärken, oder zu ersetzen, schwinden kann. Mit anderen Worten: die Wachstumsregulierung der Zelle ist bis zu einem gewissen Grade unabhängig von der Empfindlichkeit derselben gegen Reize. Es ist denkbar, dass eine Zelle schwer zur Wucherung zu bringen ist, danu aber nach Ueberwindung der vorhandenen Widerstände ihr Wachstum nicht wieder zu hemmen vermag, dass sie auf diese Weise zur Malignität "disponiert" sein kamn, ohne bereits bösartig wuchern zu müssen.

In den geschilderten Experimenten liegt nun der Nachweis vor, dass es möglich ist, auch bei normalen Körperzellen die wichtigste Eigenschaft der Zellen maligner Tumoren, die Herabsetzung des Selbsthemmungsvermögens, wenn auch nur in ganz bescheidenem Umfange, hervorzurufen. Es ist somit prinzipiell denkbar, dass durch äussere Reize die Körperzellen in der Weise verändert werden, wie wir es bei der Genese der Malignität unbedingt voraussetzen müssen.

Dr. Richard Lewis obn (Heidelberg):

\title{
Zur Behandlung maligner Tumoren mit Röntgenstrahlen.
}

Meine Herren! Ich möchte mir erlauben, Ihnen in aller Külze über die Erfahrungen zu berichten, die wir an der Czernyschen Klinik mit 
100 Internat. Konf. f. Krebsforschung, Sept. 1906 zu Heidelberg u. Frankfurt a. M.

der Röntgentherapie maligner Tumoren gemacht haben. Der Versuch, maligne Neubildungen mit X-Strahlen zu behandeln, ist zuerst in den Jahren 1900 und 1901 gemacht worden, während therapeutische Versuche an Lupus, Favus, Sycosis usw. bereits seit dem Jahre 1897 angestellt wurden. Während diese letzteren Hautaffektionen zum Teil zu glänzenden Heilungsresultaten führten und inzwischen eine sichere Domäne des Röntgenologen geworden sind, lassen die Erfolge bei malignen Tumoren immer noch sehr zu wünschen übrig, wenigstens wenn man die oft in den einschlägigen Berichten mit aufgeführten Fälle von Llcus rodens ausscheidet. Das Ulcus rodens ist, wenn es auch histologisch als Carcinom $\mathrm{zu}$ bezeichnen ist, klinisch, speziell was die Malignität anlangt, keineswegs immer auf ein und dieselbe Stufe mit den übrigen Carcinomen zu stellen (ebenso wie die Epulis klinisch von den übrigen Sarkomen getrennt werden muss). Kleine Ulcera rodentia sind zwar, das hat man oft eindeatig beobachtet, mit Röntgenstrahlen ebenso wie auch mit Radium, vollkommen zum Schwinden zu bringen, aber sie wurden schon vor der Röntgenära schneller und einfacher durch Messer, Thermokauter und Caustica dauernd beseitigt.

Es gibt wohl keine Carcinom- oder Sarkomlokalisation und form, die nicht röntgenologisch in Angriff genommen wäre. In kurzer Zeit, nach mehreren kasuistischen Heilungsberichten, bildete sich ein förmlicher Enthusiasmus für diese Behandlungsmethode aus und man versuchte sein Glück nicht nur an oberfläcblichen, sondern auch an intraabdominalen Tumoren. Die Ernüchterung liess allerdings nicht lange auf sich warten, sah man doch z. B. nach anfänglichem Scbwund der Tumoren später doch nur ein um so rapideres Wachstum usw., Fragen, auf die ich später noch kurz zurückkommen werde.

Die Röntgentherapie maligner Tumoren wurde an der Heidelberger chirurgischen Klinik in den Jahren 1903-05 an 59 Fällen versucht. Die Erfolge, die wir an diesem Material erzielt haben, sind nun leider keineswegs sehr ermutigend, denn unter unsern sämtlichen Fällen befindet sich leider kein einziger, bei dem man von radikaler Heilung oder dauernder Besserung reden könnte. Das liegt entschieden teilweise an der Beschaffenheit unseres klinischen Materials, das überhaupt einen ausserordentlich hoben Prozentsatz schwerer Fälle aufweist, was zum Teil darin seinen Grund haben dürfte, dass dasselbe der Klinik zum weitaus grössten Teil von auswärts überwiesen wird. Auf demselben Umstand basiert dann auch die für eine solche Statistik nicht förderliche Tatsache, dass ein grösserer Teil der Patienten (nämlich 25) nach wenigen Sitzungen wieder ausschieden, da zur klinischen Behandlung vielen Patienten Geduld und Mittel fehlten, eine ambulante Behandlung aber wegen der grossen Entfernung des Wohnsitzes nicht möglich war. 
Unter den 34 Fällen, die längere Zeit bestrahlt wurden und deren weiterer Verlauf beobachtet werden konnte, befinden sich 11 Fälle von Hammacarcinom, 5 Fälle von Knochensarkom, 5 Fälle von Lymphosarkom, 5 Epitheliome, 4 Melanosarkomfälle, 2 Fälle von Magen-Darmcarcinom und je 1 Fall von malignem Zungen- bzw. Parotistumor. - Ich muss es mir natürlich versagen, bei der Kürze der Zeit auf die einzelnen Fälle einzugehen und mich auf eine zusammenfassende Uebersicht beschränken.

Die Fälle von Mammacarcinom waren fast ausschliesslich Recidivälle nach vorausgegangener Amputatio mammae; nur 2 waren früher noch nicht operiert worden, sie waren aber operativ nicht mebr angreifbar (in 1 Fall handelte es sich um eine 83jährige Fran mit doppelseitigem exulzeriertem Carcinom, im andern um ein ausgedehntes Carcinoma lenticulare). Ein dauernder Nutzen wurde in keinem Falle erzielt. Bei mehreren Fällen ging allerdings die Geschwulst zurück, die einzelnen Knoten schrumpften und zeigten im Zentrum etwas Erweichung. Dafür mussten wir leider öfters die Erfahrung machen, dass das Weiterwachsen des Tumors an der Peripherie viel rascher, als vor der Bestrahlung fortschritt. Dagegen erreichten wir in fast sämtlichen Fällen einen subjektiven Erfolg - die lanzinierenden, oft sehr heftigen Schmerzen der Patienten liessen meist nach wenigen Sitzungen nach; leider stellten sie sich aber nach Aussetzen der Bestrahlungen stets wieder ein. Ein weiterer Nutzen war bei den ulzerierten Tumoren insofern zu bemerken, als das für die Patientinnen so lästige Nässen nachliess und eine ziemlich rasch fortschreitende Ueberhäutung der Ulzerationen vom Rande her einsetzte. In 8 Fällen trat der Tod in Jahresfrist ein, und wir hatten leider nicht den Eindruck, als ob der Exitus durch die Röntgenbehandlung wesentlich verzögert worden wäre.

Unter den 5 Fällen von Knochensarkomen befindet sich der so hochinteressante Fall von Oberkiefersarkom, der im Laufe der Jahre 1901 bis $19045 \mathrm{mal}$ operiert wurde, seither rezidivfrei ist, und über den Ibnen Exzellenz Czerny bereits berichtet hat. Dieser Fall wurde nach der 4. Operation längere Zeit (21 Sitzungen) mit Röntgenstrahlen behandelt. Zwar wurde durch die Röntgentherapie eine gewisse Zusammenziehung und Abkapselung des Tumors erreicht, doch wurde trotzdem später eine erneute Operation notwendig und die seitherige Rezidivfreiheit ist sicher auf Konto des Messers zu setzen. - In einem weiteren Fall von ausgedehntem exulzerierten Oberkiefersarkom wurde mehrere Monate hindurch (natürlicb mit den notwendigen Pausen) Röntgentherapie ohne Nutzen angewandt. Patient starb 2 Monate nach der als nutzlos ausgesetzten X-Strahlenbehandlung. - Ebenfalls ohne Nutzen wurden 3 Fälle von Sarkom der oberen Extremität behandelt, sie kamen alle zum Exitus. In 1 Fall musste successive vom Vorderarm bis zur Scapula und Clavicula 
die ganze Extremität wegen Rezidivs entfernt werden, obgleich jeweils nach den einzelnen Operationen prophylaktische Bestrahlungen vorgenommen waren.

Bei 3 Fällep von Lymphosarcoma colli (davon der eine allerdings schon mit Bauchmetastasen) konnte auch nur eine Linderung der Schmerzen und ein vorübergehendes Zusammenschmelzen der Knoten erreicht werden; alle drei waren bei einer vor einigen Monaten ergangenen Umfrage nach den Schicksalen der Patienten bereits verstorben. Ausserdem fielen unter diese Gruppe 2 Fälle von Mediastinaltumoren, die vorübergehend, aber auch nur vorübergehend, subjektiv und objektiv (Rückgang des Dämpfungsbezirks, Abnahme der Dyspnoe usw.) entschieden gebessert wurden. Die Lymphosarkome sind ja entschieden Tumorarten, die auf Röntgenstrahlen ziemlich rasch reagieren und es sind $j a$ auch in der Literatur Fälle veröffentlicht worden, wo in der Tat ein rapider Rückgang der Geschwulst beobachtet wurde; ob in diesen Fällen wirklich Dauerheilungen vorliegen, erscbeint einstweilen allerdings noch keineswegs sicher, da die Beobachtungszeit noch zu kurz ist.

Wie vorsichtig man in der Bewertung scheinbarer Röntgenerfolge sein muss, zeigt ein Fall der Gruppe IV: Epitheliome. Es handelt sich um eine 70jährige Frau, über die mein Vorgänger in der Leitung unseres Röntgen-Laboratoriums, Herr Dr. Engelken, im Jahresbericht unserer Klinik von 1904 berichten konnte, dass nach wenigen Sitzungen das anfangs fünfmarkstückgrosse Ulcus grossenteils mit schöner, glatter, solider Haut bedeckt war. Die mikroskopische Untersuchung einer Randpartie ergab typische Degenerationserscheinungen (Quellung, Einschmelzung, Vakuolenbildung), der Fall schien also prognostisch sehr günstig. Trotzdem war die Heilung nur eine unvollkommene. Die Patientin steht seit über Jahresfrist wieder in unserer Behandlung und trotz Versuchen mit verschiedenen Methoden (Röntgen, Chromätzungen usw.) greift das Epitheliom zwar langsam, aber stetig weiter. Von einer Operation wollte die Patientin früher nichts wissen, jetzt ist das Carcinom auf den Schädel übergegriffen und inoperabel. Aebnlich ging es uns mit 4 weiteren Fällen von Ohr- bzw. Gesichtsepitheliom, die auch nur vorübergehend Besserung zeigten. Wir konnten uns hier, wie auch bei anderen Röntgenfällen, des Eindrucks nicht erwehren, dass die Krebszellen anfänglich durch die Röntgenstrahlen in ihrem Wachstum stark behindert wurden, dass es ibnen aber nach einer gewissen Zeit gelang, auch über diese (von ihrem Standpunkt aus betrachtete) Noxe Herr zu werden, so dass sie, man möchte sagen ungestört trotz der Bestrahlung weiterwachsen und sich vermehren konnten.

Vollständig versagt hat die Röntgentherapie bei 4 Fällen von Melanosarkomen, die sämtlich nach einigen Monaten zum Exitus kamen, ebenso bei 2 Fällen von intrabdominellen Carcinomen, bei denen die Behandlung eigentlich auch nur solaminis causa versucht wurde. Ebenfalls vergeblich 
war der Versuch, ein Zungencarcinom bei einem alten Herrn von 75 Jahren und einen Parotistumor mit Röntgenstrahlen zu bessern.

Gestatten Sie mir jetzt einige kurze Worte über die Technik; genauer auf dieselbe einzugehen würde viel zu weit führen. Wir sind im allgemeinen so verfahren, dass wir 5 Tage nacheinander je 10-15 Minuten in einer I)istanz von $15-25 \mathrm{~cm}$ mit mittelweicher bis harter Röhre (dem speziellen Fall und dem zu erreichenden Resultat angepasst) bestrahlten und dann 8 Tage bis zu einem neuen Turnus pausierten. Natürlich ist das nur ein allgemeines Schema und wir passten uns dem Verlauf der Fälle mit unserer Mlethodik an, wie ja überhaupt gerade bei der Röntgentechnik ein feines Individualisieren von grösster Bedeutung ist. Sahen wir z. B., wie das öfters der Fall war, bereits nach wenigen Sitzungen eine Rötung der Haut auftreten, so setzten wir natürlich sofort mit der Behandlung aus. Richtige Dermatitiden haben wir nur ganz im Anfang unserer therapeutischen Röntgenversuche auftreten sehen, als wir noch 25 Minuten bestrablten, zu einer Zeit, wo die Gefahren dieser Therapie noch nicbt so bekannt waren, wie heutzıtage.

Das Holzknechtsche Chromoradiometer zur quantimetrischen Messung der verabfolgten Dose haben wir sehr begrüsst und sofort in Gebrauch genommen, da uns bis dahin ein Massstab, der ja speziell auch für den Vergleich mit Resultaten anderer Autoren von grösster Bedeutung ist, fehlte. Es ist Ihnen ja bekannt, dass das Chromoradiometer in seiner jetzigen Gestalt leider nicht für längere Zeit verwendbar ist. Auch die anderen Methoden (Freund, Kienböck usw.) haben sich bisher nicht eingebürgert, weil sie für den täglichen Gebrauch noch nicht einfach genug, oder nicht einwandsfrei sind. Erst in letzter Zeit bedienten wir uns der Walterschen Messmethoden, die ja einen wesentlichen Fortschritt für die Frage der Dosierung zu bedeuten scheinen. - Die gesamte Bestrahlungszeit schwankte bei unseren Fällen zwischen $11 / 2$ und 8 Stunden.

Wenn wir uns die Frage vorlegen, was wir in unseren Fällen mit der Therapie erreicht haben, so war nur ein Moment, was wir in den weitaus meisten Fällen erreichen konnten, und was ja für die armen Patienten von grossem Wert ist, das ist die analgesierende Wirkung der Röntgenstrahlen. Ausserdem versiegte in fast allen Fällen, wo Ulzerationen bestanden, meist schon nach wenigen Sitzungen das so lästige Nässen. Dass wir öfters lokal einen Rückgang der Knoten konstatieren konnten, wurde bereits eingangs erwähnt. Wir konnten uns aber oft des Eindrucks nicht erwehren, als ob in zeitlichem Zusammenhang mit diesen Rückbildungen das Fortwuchern an der Peripherie und die Neigung zur Metastasierung zunahm. Die Möglichkeit, dass durch die Röntgenbestrahlung den Abwehrversuchen des Körpers gegen das Umsichgreifen des Tumors, i. e. Abkapselungsprozessen, entgegengearbeitet und dass durch die herbeigeführte Erweichung 
104 Internat. Konf. f. Krebsforschung, Sept. 1906 zu Heidelberg u. Frankfurt a. M.

die Verschleppung von Tumormaterial vielleicht begünstigt wird, ist jedenfalls nicht absolut von der Hand zu weisen.

Die bis jetzt in der Literatur niedergelegten Berichte von Heilung maligner Tumoren durch Röntgentherapie halten grösstenteils einer scharfen Kritik nicht Stand. Es werden eben oft vorübergehend gebesserte Fälle publiziert, bzw. in medizinischen Vereinen vorgestellt, ohne dass die betreffenden Autoren nochmals über die betreffenden Fälle berichten, wenn sie nach Monaten wieder ein viel schlechteres Bild liefern; damit erscheint natürlich der wirkliche Stand der Röntgentherapie in unrichtigem Licht. Es ist ja nicht zu leugnen, dass vereinzelte Röntgenerfolge berichtet sind, so z. B. unlängst von Kienböck bei einem grossen Mediastinalsarkom. Aber der Fall liegt noch nicht weit genug zurück, um von Dauerheilung reden zu können, speziell da man gerade bei Sarkomen, wie das auch Coley betont hat, leider nur zu oft Spätrezidive antrifft. Bei den von Lassar in der Berliner medizinischen Gesellschaft 1904 und 1905 rorgestellten Fällen (Mammacarcinome usw.) konnte, wie v. Bergmann sofort in der Diskussion hervorbob, von Heilung keine Rede sein. Man hat auch schon vor der Röntgenära mehr oder weniger grosse Tumorknoten spontan verschwinden sehen, und es ist daher oft schwer zu entscheiden, wie viel bei den einzelnen Fällen von Tumorrückbildung wirklich auf Kosten des Röntgenlichtes zu setzen ist.

Wenn ich unsere Resultate zum Schluss nochmals kurz zusammenfassen darf, so konnten wir bei unseren Fällen einen dauernden Erfolg nicht erreichen. Neben sehr passageren Besserungen erzielten wir durch die Bestrahlungen nur ein Nachlassen der Schmerzen und bei ulzerösen Tumorformen ein Versiegen der Sekretion. Ich habe bereits eingangs betont, dass unser Material ein sehr schweres war. So wird an einer chirurgischen Klinik das Material wohl immer mehr oder weniger beschaffen sein, denn für operable Tumorfälle ist das Messer einstweilen immer noch die beste Hülfe. Wir haben uns aber für verpflichtet gebalten, unser Iaterial Ihnen vorzulegen, da es, im Verein mit mehreren in letzter Zeit erschienenen Publikationen anderer Institute dazu dienen könnte, vor zu hochgestellten Erwartungen auf die Röntgentherapie zu bewahren, was ja der Methode nur dienlich sein kann. Wie die Röntgentberapie jetzt gehandhabt wird, dürften keine zu grossen Hoffnungen an sie zu knüpfen sein (wegen der geringen Tiefenwirkung usw.). Es werden ja von verschiedensten Seiten Versuche zur Verbesserung des therapeutischen Effekts des Röntgenlichtes gemacht, und es wäre natürlich mit Freuden zu begrüssen, wenn diese Versuche dahin führen sollten, dass uns damit ein wirkliches Heilmittel gegen die malignen Tumoren in die Hand gegeben würde.

(Schluss der Sitzung 21/2 Uhr.) 